\title{
Initiation Mechanism of the Copolymerization of Carbon Dioxide and Propylene Oxide with Zinc Carboxylate Catalyst Systems
}

\author{
Yoshihiro Hino, Yasuhiko YoshidA, and Shohei INOUE \\ Department of Synthetic Chemistry, Faculty of Engineering, \\ University of Tokyo, Hongo, Bunkyo-ku, Tokyo 113, Japan
}

(Received October 19, 1983)

\begin{abstract}
The mechanism for the copolymerization of carbon dioxide and propylene oxide using diethylzinc--benzenedicarboxylic acid and zinc oxide-benzenedicarboxylic acid systems as catalysts was investigated. The aromatic residue originating from the catalyst systems was confirmed to combine with the copolymer chain by gel permeation chromatographic studies. From the molar concentration of the aromatic residue per weight of the copolymer and the number average molecular weight, one aromatic residue was found to be incorporated into each copolymer chain when diethylzinc-based systems were used as catalysts. Thus, the copolymerization of carbon dioxide and propylene oxide is considered to be initiated by the insertion of a monomer into a zinccarboxylate bond of the catalysts.
\end{abstract}

KEY WORDS Propylene Oxide / Copolymerization / Carbon Dioxide / Poly(propylene carbonate) / Diethylzinc / Zinc Oxide /

The copolymerization of carbon dioxide and epoxide using organozinc catalyst systems has been studied extensively. ${ }^{1}$ Diethylzinc-dihydric phenol ${ }^{2,3}$ or diethylzinc-benzenedicarboxylic acid system ${ }^{4}$ was found to be a very effective catalyst for the copolymerization. A system based on an inorganic zinc compound such as zinc oxide ${ }^{5}$ or zinc dihydroxide $^{6}$ and a carboxylic acid was also found effective. In the diethylzinc-dihydric phenol system, the mechanism involving initiation by insertion of a monomer into the zincphenolate bond was proposed on the basis of the agreement between the number average molecular weight of the copolymer, calculated by the concentration of resorcinol residues incorporated into the copolymer determined by UV spectroscopy and that measured by vapor pressure osmometry (VPO). ${ }^{3}$ In this study, studies of the copolymer obtained by diethylzinc-benzenedicarboxylic acid systems and zinc oxide-isophthalic acid-propionic acid system by using gel permeation chromatography (GPC) were carried out to confirm that residues originated from the catalyst systems combine with the polymer chain.

\section{EXPERIMENTAL}

\section{Materials}

Propylene oxide was distilled over potassium hydroxide and then over calcium hydride in nitrogen. Carbon dioxide was used directly from a cylinder.

Commercially available dioxane was added by dilute hydrochloric acid and this mixture was refluxed for $12 \mathrm{~h}$ while a nitrogen stream was bubbled through it. Excess potassium hydroxide was then added and the flask was shaken well to neutralize the mixture. The upper layer was separated and distilled over potassium hydroxide and then over sodium metal in nitrogen.

Diethylzinc was distilled under reduced 
pressure in a nitrogen atmosphere, bp $45^{\circ} \mathrm{C} /$ $40 \mathrm{mmHg}$. Commercially available zinc oxide was used without further purification. Isophthalic acid was purified by recrystallization from ethanol. Terephthalic acid was allowed to react with an equimolar amount of sodium carbonate in water to form sodium terephthalate, which was purified by recrystallization from water. The salt was dissolved in water and dilute hydrochloric acid was added to liberate terephthalic acid which was then filtered, washed with water, and dried under reduced pressure. Resorcinol was purified by recrystallization from toluene.

\section{Preparation of Catalysts}

Diethylzinc System. A solution of isophthalic acid $(1.18 \mathrm{~g}, 7.3 \mathrm{mmol})$ (or terephthalic acid or resorcinol) in dioxane $\left(30 \mathrm{~cm}^{3}\right)$ was slowly added dropwise (ca. $25 \mathrm{~min}$.) to a solution of diethylzinc $\left(0.8 \mathrm{~cm}^{3}, 7.8 \mathrm{mmol}\right)$ in dioxane $\left(20 \mathrm{~cm}^{3}\right)$ with stirring, and the mixture was stirred for $3 \mathrm{~h}$ to give a white emulsion. This procedure had to be performed in a nitrogen atmosphere. The emulsion thus obtained was used as a catalyst and transferred by a syringe to an autoclave for polymerization.

Zinc Oxide System. ${ }^{5}$ A mixture of zinc oxide $(1.22 \mathrm{~g}, 15 \mathrm{mmol})$ and isophthalic acid $(1.25 \mathrm{~g}$, $7.5 \mathrm{mmol})$ in dioxane $\left(120 \mathrm{~cm}^{3}\right)$ was refluxed with stirring for $2 \mathrm{~h}$ in nitrogen. Following the addition of propionic acid $\left(1.1 \mathrm{~cm}^{3}, 15 \mathrm{mmol}\right)$, the mixture was refluxed for $3 \mathrm{~h}$. The solvent was removed under reduced pressure at $120^{\circ} \mathrm{C}$ to leave a white powdery solid, $1.20 \mathrm{~g}$ of which was used as the catalyst.

\section{Polymerization}

The catalyst and propylene oxide $(17.2 \mathrm{~g}$, $0.296 \mathrm{~mol}$ ) were placed in a stainless autoclave, in which the atmosphere had been replaced with carbon dioxide, and carbon dioxide was introduced directly from a cylinder ( $c a$. $40 \mathrm{~atm})$. The reaction mixture was stirred for $40 \mathrm{~h}$ at $35^{\circ} \mathrm{C}$. The reaction mixture was then diluted with benzene, and washed successively with dilute hydrochloric acid, water, and an aq. $\mathrm{NaHCO}_{3}$ solution. The benzene layer was poured into methanol to precipitate the copolymer, poly(propylene carbonate).

\section{Measurements}

The number average molecular weight of the copolymer was measured at $50.9^{\circ} \mathrm{C}$ in benzene with a CORONA 117 vapor pressure osmometer (VPO). Gel permeation chromatography (GPC) of the copolymer was carried out in tetrahydrofuran (THF) using a TOYO SODA HLC-802A. When detection was made by UV absorption, the wavelength was adjusted to that of the absorption maximum by aromatic groups present in the catalyst system. The curve obtained by the refractive index method was calibrated with polystyrene standards so as to calculate the molecular weight distribution curve in terms of molar ratio. The UV absorption spectrum of the copolymer was measured in chloroform $(2 \mathrm{wt} \%)$ at room temperature on JASCO UVIDEC-1, and compared with that of model compounds (dimethyl isophthalate, dimethyl terephthalate, and 1,3-dimethoxybenzene) to determine the number of aromatic residues in the copolymer, assuming that the molar extinction coefficients were the same for the aromatic group in the copolymer and for that in the corresponding model compound.

\section{RESULTS AND DISCUSSION}

The results of the copolymerization using various catalyst systems are shown in Table I including a diethylzinc-resorcinol one.

It has been suggested that the diethylzincbenzenedicarboxylic acid, ${ }^{4}$ zinc oxide-isophthalic acid-propionic acid $(2: 1: 2),{ }^{5}$ and diethylzinc-resorcinol ${ }^{3}$ systems have the structures 1, 2, and 3, respectively.

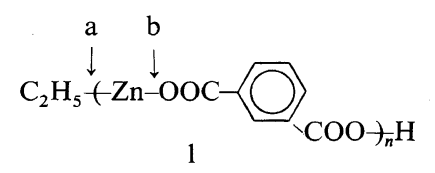

Polymer J., Vol. 16, No. 2, 1984 
Table I. Copolymerization of carbon dioxide and propylene oxide (PO)

\begin{tabular}{|c|c|c|c|c|c|}
\hline No. & Catalyst & & Time & Yield & $10^{-3} \times M_{n}^{\mathrm{b}}$ \\
\hline 1 & Diethylzinc-isophthalic acid & $(1: 0.9)$ & 39 & 6.6 & 74 \\
\hline 2 & Diethylzinc-terephthalic acid & $(1: 0.9)$ & 37 & 1.3 & 65 \\
\hline $3^{c}$ & Zinc oxide-isophthalic acid-propionic acid & $(2: 1: 2)$ & $>30$ & 9.3 & 31 \\
\hline 4 & Diethylzinc-resorcinol & $(1: 0.9)$ & 40 & 6.0 & 56 \\
\hline
\end{tabular}

${ }^{\text {a }} \mathrm{PO}, 17.2 \mathrm{~g} ; \mathrm{CO}_{2}, 40 \mathrm{~kg} \mathrm{~cm}^{-2}$; dioxane, $50 \mathrm{~cm}^{3}$; reaction temp, $35^{\circ} \mathrm{C} ;[\mathrm{Zn}] /[\mathrm{PO}]_{0}=2.6 \mathrm{~mol} \%$.

b Measured by VPO in a benzene solution at $50.9^{\circ} \mathrm{C}$.

c Bulk polymerization at $80^{\circ} \mathrm{C} ;[\mathrm{Zn}] /[\mathrm{PO}]_{0}=2.3 \mathrm{~mol} \%$.

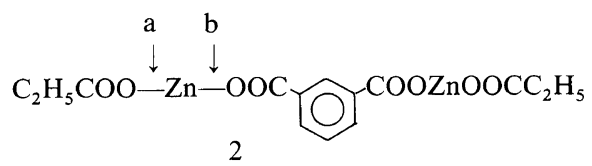

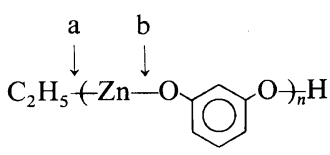

3

In each catalyst system, there are two different active sites, which possibly participate in the polymerization reaction, as indicated by arrows in each formula. If the copolymerization reaction is initiated at the " $b$ " site of the respective catalyst systems, an aromatic group should be incorporated into a chain end of the copolymer. However, the reaction at the "a" site does not result in the incorporation of an aromatic group into the product.

\section{Gel Permeation Chromatographic Studies}

The GPC chart of the copolymer of carbon dioxide and propylene oxide obtained by the diethylzinc-isophthalic acid $(1: 1)$ system is shown in Figure 1. The UV absorption observed (upper solid line) indicates that the aromatic group (from isophthalic acid) contained in the catalyst system is incorporated into the polymer chain, since poly(propylene carbonate) itself has no absorption within the region examined $(250-300 \mathrm{~nm}) .^{3}$ The lower solid line in this chart was obtained by the refractive index (RI) method and shows the
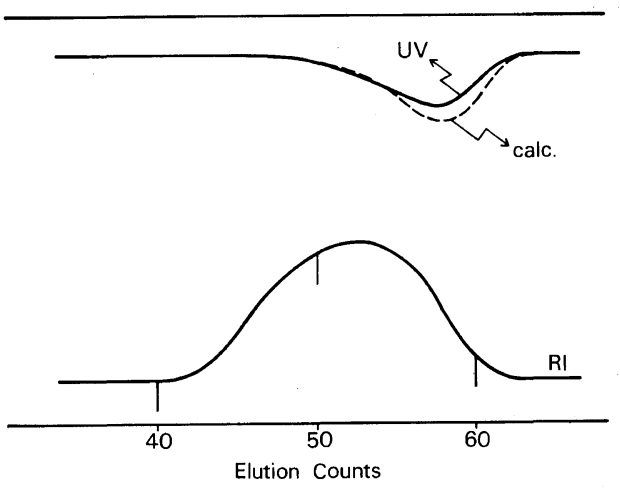

Figure 1. GPC curves of the copolymer of carbon dioxide and propylene oxide obtained with the diethylzinc-isophthalic acid system, measured in THF. calc., the curve obtained by calibration of the RI curve with polystyrene standards, representing the distribution of the number of copolymer molecules with respect to the molecular size.

weight distribution of the copolymer molecules with respect to molecular size. The broken line corresponds to the calculated molecular weight distribution curve as to the number of copolymer molecules, and was obtained by dividing the value for the RI curve by the molecular weight determined by the calibration curve using polystyrene standards at the corresponding elution counts.

The good agreement obtained between the calculated curve and that by UV absorption indicates that a constant number of aromatic residues from isophthalic acid is incorporated into one polymer chain. Similar results were 


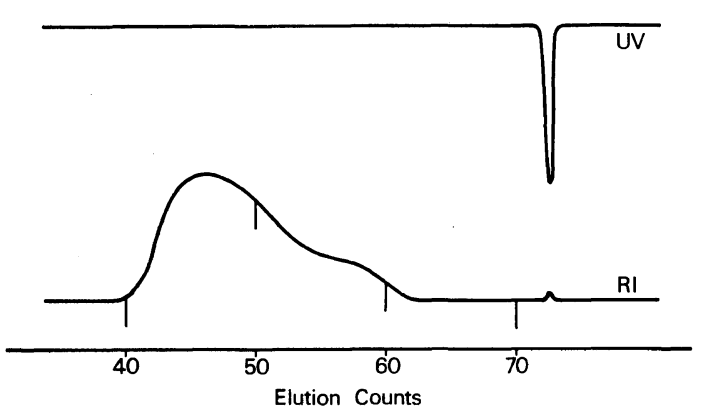

Figure 2. GPC curves of a mixture of dimethyl isophthalate and the copolymer obtained in the diethylzinc-water system.

obtained for diethylzinc-terephthalic acid (1:1), zinc oxide-isophthalic acid-propionic acid $(2: 1: 2)$, and diethylzinc-resorcinol $(1: 1)$ systems.

To confirm that the residue from isophthalic acid combined chemically with the polymer chain, dimethyl isophthalate was dissolved and stirred well in a THF solution of poly(propylene carbonate) separately prepared by the diethylzinc-water $(1: 1)$ system but we showed no absorption at $280 \mathrm{~nm}$ ( $\lambda_{\max }$ of isophthalic acid). When this solution was subjected to GPC, dimethyl isophthalate was found to be eluted in a fraction different from the copolymer (Figure 2). Thus, the UV absorption in GPC is considered to arise from the aromatic residue combined chemically with the polymer chain.

\section{Number of Aromatic Residues per Copolymer Molecule}

For a quantitative determination of aromatic residues per copolymer molecule, the UV absorption was compared with that of model compounds such as dimethyl isophthalate, dimethyl terephthalate, and 1,3-dimethoxybenzene, corresponding to the aromatic groups considered to be incorporated into the copolymer obtained by the diethylzinc-isophthalic acid $(1: 1)$, diethylzincterephthalic acid $(1: 1)$, zinc oxide-isophthalic
Table II. Concentration $C$ of aromatic residues in the copolymer and the number $N$ in a polymer chain

\begin{tabular}{lrrr}
\hline \multicolumn{1}{c}{ Catalyst } & \multicolumn{1}{c}{$C$} & \\
\cline { 3 - 3 } & & $\mathrm{mol} \mathrm{g}^{-1}$-polym. & \\
\hline $\begin{array}{l}\text { Diethylzinc-isophthalic } \\
\text { acid }\end{array}$ & $(1: 0.9)$ & $1.4 \times 10^{-5}$ & 1.0 \\
$\begin{array}{l}\text { Diethylzinc-terephthalic } \\
\text { acid }\end{array}$ & $(1: 0.9)$ & $1.8 \times 10^{-5}$ & 1.2 \\
$\begin{array}{l}\text { Zinc oxide-isophthalic } \\
\text { acid-propionic acid }\end{array}$ & $(2: 1: 2)$ & $2.4 \times 10^{-5}$ & 0.7 \\
$\begin{array}{c}\text { Diethylzinc-resorcinol } \\
(1: 0.9)\end{array}$ & $1.8 \times 10^{-5}$ & 1.0 \\
\hline
\end{tabular}

acid-propionic acid $(2: 1: 2)$, and diethylzincresorcinol (1:1) systems, respectively. Concentration of the aromatic residues in the copolymer, $C$ ( $\mathrm{mol} / \mathrm{g}$-polymer), was determined from the absorption intensity of the copolymer and the molar extinction coefficients of the corresponding model compound by using the following equation.

$$
\frac{A}{\varepsilon \cdot l}=C
$$

where $A$ represents the absorption intensity of the polymer solution having a concentration $1 \mathrm{gl}^{-1}$ at $\lambda_{\max }\left(\mathrm{g}^{-1}\right), \varepsilon$, molar extinction coefficient $\left(1 \mathrm{~mol}^{-1} \mathrm{~cm}^{-1}\right)$, and $l$, cell length $(\mathrm{cm})$. The product $(N)$ of the number average molecular weight of the copolymer and molar concentration $(C)$ of the aromatic residues per weight of copolymer corresponds to the number of aromatic residues in one polymer chain. The calculated values are summarized in Table II. As seen in Table II, one aromatic residue was incorporated into each polymer chain when the diethylzinc-based system was used as the catalyst. Therefore, the copolymerization is considered to be initiated almost exclusively at the " $b$ " site in formulas 1 and 3 .

The results obtained in the present study with the diethylzinc-resorcinol system confirms the conclusions of our previous paper. ${ }^{3}$ In the case of the zinc oxide-isophthalic acidpropionic acid system, copolymerization is 
considered to be initiated also at the " $a$ " site in formula 2 .

\section{REFERENCES}

1. For a review: S. Inoue and N. Yamazaki, Ed., "Organic and Bioorganic Chemistry of Carbon Dioxide," Kodansha, Tokyo, 1981, p 167.
2. M. Kobayashi, S. Inoue, and T. Tsuruta, Macromolecules, 4, 658 (1971).

3. M. Kobayashi, Y. Tang, T. Tsuruta, and S. Inoue, Makromol. Chem., 169, 69 (1973).

4. M. Kobayashi, S. Inoue, and T. Tsuruta, J. Polym. Sci., Polym. Chem. Ed., 11, 2383 (1973).

5. S. Inoue, T. Takada, and H. Tatsu, Makromol. Chem., Rapid Commun., 1, 775 (1980).

6. K. Soga, E. Imai, and I. Hattori, Polym. J., 13, 407 (1981). 